\title{
Inflammation and reduced endothelial function in the course of severe acute heart failure
}

\author{
STIG E. HERMANSEN, TRINE KALSTAD, OLE-JAKOB HOW, and TRULS MYRMEL
}

TROMS $\varnothing$, NORWAY

\begin{abstract}
Systemic inflammation and elevated circulating levels of the endogenous nitric oxide inhibitor asymmetrical dimethylarginine (ADMA) have been associated with increased risk in cardiogenic shock (CS). In this prospective study, we assessed, over 4 consecutive days, the changes and possible associations between vascular function, markers of inflammation, and circulating ADMA levels in patients with CS $(n=12)$ and postcardiotomy heart failure $(n=12, P C-H F)$. Vasodilator function was measured as a reactive hyperemia index ( $\mathrm{RH}$-index) using a finger plethysmograph. Blood samples were analyzed for plasma ADMA, interleukine-6, interleukine-8, intracellular adhesion molecule-1, and vascular adhesion molecule-1. Baseline RH-index was significantly attenuated compared with healthy controls (2.28) for both CS and PC-HF ( 1.35 and 1.45 , respectively, $P=.001$ ). Although vasodilator function improved in PC-HF patients, it remained attenuated in CS. Inflammatory markers were markedly elevated followed by a significant fall during the observation period in both groups. ADMA levels increased significantly during the observation period for $\mathrm{PC}-\mathrm{HF}$, whereas no pattern of change was observed for CS. No association was found between the longitudinal changes in RH-index, markers of inflammation, or ADMA in CS. However, an improved RH-index was associated with decreasing inflammatory markers in PC-HF. ADMA correlated to arterial lactate levels and the degree of organ dysfunction in CS. In conclusion, CS and PC-HF were characterized by a marked inflammatory activation accompanied by an attenuated vasodilator function. ADMA was related to organ dysfunction and degree of hypoperfusion during CS but showed no correlations to inflammation or hampered vasodilator function. The pathogenic significance of these responses needs clarification. (Translational Research 2011; $\mathbb{0}: 1-11$ )
\end{abstract}

\begin{abstract}
Abbreviations: $A D M A=$ asymmetrical dimethylarginine; $C A B G=$ coronary artery bypass grafting; $\mathrm{CCU}=$ coronary care unit; $\mathrm{CPR}=$ cardiopulmonary resuscitation; $\mathrm{CS}=$ cardiogenic shock; $\mathrm{DDAH}=$ dimethylarginine dimethylaminohydrolase; $\mathrm{HR}=$ heart rate; IABP = intra-aortic balloon pump; iCAM-1 = intracellular adhesion molecule- $1 ; \mathrm{ICU}=$ intensive care unit; IL-6 = interleukine 6; IL-8 = interleukine 8; $I Q R=$ interquartile range; IS = inotropic score; MAP = mean arterial pressure; $\mathrm{NO}=$ nitric oxide; $\mathrm{PC}-\mathrm{HF}=$ postcardiotomy heart failure; $\mathrm{RH}$-index = reactive hyperemia index; SOFA = Sequential Organ Failure Assessment; TNF $\alpha=$ tumor necrosis factor $\alpha$; vCAM-1 = vascular cell adhesion molecule-1
\end{abstract}

From the Department of Cardiothoracic and Vascular Surgery, University Hospital of North Norway; and Department of Clinical Medicine; Department of Medical Biology, The Health Faculty, University of Troms $\varnothing$, Troms $\varnothing$, Norway.

Supported by Helse Nord RHF.

Submitted for publication June 30, 2010; revision submitted December 5, 2010; accepted for publication December 10, 2010.
Reprint requests: Stig Eggen Hermansen, Department of Cardiothoracic and Vascular Surgery, University Hospital of North Norway, 9038 Troms $\varnothing$, Norway; e-mail: stig.eggen.hermansen@unn.no.

$1931-5244 / \$$ - see front matter

(c) 2011 Mosby, Inc. All rights reserved.

doi:10.1016/j.trsl.2010.12.002 


\section{AT A GLANCE COMMENTARY}

\section{Hermansen S, et al.}

Background

Systemic inflammation and elevated levels of the endogenous NO-inhibitor asymmetrical dimethylarginine (ADMA) are associated with poor outcomes in acute severe heart failure. How these risk markers relate to each other and their significance in the pathophysiology and vascular dysfunction in the course of cardiogenic shock (CS) is, however, unknown.

\section{Translational Significance}

We observed a marked inflammatory response and attenuated vasodilator function in the acute phase of CS. ADMA correlated to the degree of hypoperfusion and organ dysfunction. A substantial degree of individual and time-related fluctuations in these parameters demonstrates the challenges in clarifying the pathophysiological mechanisms in the circulatory dysfunction during CS.

Recent clinical trials and registries on patients with cardiogenic shock (CS) have demonstrated the heterogeneity of this severely ill patient population with regard to etiology as well as clinical and hemodynamical presentation. ${ }^{1-4}$ The current understanding of the underlying pathophysiology that marks patients with an unfavorable course and lack of response to treatment is limited. This was evident in the recently published Triumph trial that addressed the possible adverse effects of a presumed excessive nitric oxide (NO) production secondary to inflammation in CS. ${ }^{5}$ In this study, treatment with an unselective NO inhibitor in patients with persisting shock failed to reduce the duration of shock and mortality. However, observational studies have suggested that systemic inflammation and neurohormonal activation plays an important role in the pathogenesis of CS. ${ }^{3,6-10}$

The naturally occurring NO-inhibitor asymmetrical dimethylarginine (ADMA) has been implicated in the pathogenesis of endothelial dysfunction and several chronic cardiovascular disorders. ${ }^{11-14}$ In contrast to its increasing recognition as a risk marker in cardiovascular disease, little is known about ADMA's role in the acute setting. Experimental studies, however, have demonstrated adverse hemodynamic effects after systemic administration of ADMA in humans. ${ }^{15}$ Furthermore, ADMA has been indicated to be an independent predictor for a poor outcome in critical illness and CS. ${ }^{16,17}$
Elevated levels of ADMA also have been observed in patients with acute decompensated heart failure as compared with chronic heart failure. ${ }^{18}$ However, a recent study on acute heart failure failed to confirm these findings, and ADMA also did not seem to identify patients with increased risk for future cardiac events including episodes of decompensation. ${ }^{19}$ However, at this time, the biological significance and possible regulatory role of ADMA as a modulator of NO production and thus as a vascular function in acute heart failure is not known.

It is necessary to characterize and establish the different factors modulating the circulation in patients with severe pump failure. Ultimately, these observations hopefully will identify and tailor an optimal medical treatment for different subgroups of patients.

The main aim of this observational study therefore was to examine the changes and possible relations among the endogenous NO inhibitor ADMA, vascular vasodilator function and markers of inflammation, and endothelial activation during the course of severe acute heart failure (ie, CS and postcardiotomy heart failure). In addition, we assessed their association with organ dysfunction and patient outcomes.

\section{METHODS}

Study population. The study population consisted of 24 prospectively included patients admitted to the intensive care unit (ICU) or coronary care unit (CCU) at the University Hospital of North Norway between 2007 and 2009 with either CS or postcardiotomy heart failure (PC-HF). Our institution is a university hospital serving a population of approximately 500,000. Informed written consent was obtained from each patient if possible or the nearest relative. The study protocol was approved by the regional ethics committee (REK-Nord 51/2004) and conforms with the ethical guidelines outlined in the Declaration of Helsinki.

CS was defined as cardiac dysfunction with persistent hypotension (systolic blood pressure $<90 \mathrm{mmHg}$ ) or the need for vasopressor/inotropic drug support to keep systolic blood pressure greater than $90 \mathrm{mmHg}$ in combination with signs and symptoms of tissue hypoperfusion despite a correction of preload. Signs of systemic hypoperfusion included altered mental state, cool skin and extremities, low urine output $(<0.5 \mathrm{~mL} / \mathrm{kg} / \mathrm{h})$, or elevated arterial lactate. PC-HF was defined as inadequate cardiac performance after open-heart surgery in need of inotropes/vasopressors and/or mechanical circulatory support for more than $2 \mathrm{~h}$ after surgery with or without signs of systemic hypoperfusion. The PC-HF group did not include patients where an intra-aortic balloon pump (IABP) was implanted preoperatively to improve 
a critical coronary perfusion in otherwise circulatory stable patients, and this support was continued postoperatively without the need for additional inotropic support. Exclusion criteria were age $<18$ years and recent major surgery other than heart surgery. Data on prior medical history were obtained from the patients medical charts at study entry. Basic hemodynamic variables including mean arterial pressure (MAP), heart rate (HR), central venous pressure, and blood gas analyses (arterial $\mathrm{pH}$, base excess) were recorded at study inclusion in all patients and thereafter daily at the time of the vascular functional assessments. Invasive hemodynamic monitoring with pulmonary artery catheter was not obligatory in the study protocol and only applied on clinical indication. To give an estimate of the severity of circulatory compromise, the concomitant requirements of inotropic and/or vasopressor agents were presented as total vasopressor dose (epinephrine + norepinephrine) and inotropic score (IS). IS summarizes the total dosing equivalents of inotropes and vasopressors in $\mu \mathrm{g} / \mathrm{kg} / \mathrm{min}$ (dopamine + dobutamine + milrinone $\times 15+$ epinephrine $\times 100+$ norepinephrine $\times 100)^{20,21}$ The occurrence of organ dysfunction and failure, from admission through day 4, was assessed by the Sequential Organ Failure Assessment (SOFA) score. ${ }^{22}$ The maximal SOFA score was calculated based on the worst score for each organ component in this time period. The neurological subscore was not included because of the high number of patients under sedation both at onset of heart failure and during ICU/CCU treatment. A SOFA subscore $\geq 3$ was defined as organ failure.

Blood sampling and biochemical assays. Blood samples were collected in ethylenediaminetetraacetic acid containing tubes at baseline (day 1) and for the next 3 consecutive days. Plasma samples were frozen immediately after centrifugation and stored at $-70^{\circ} \mathrm{C}$ until subsequent analysis. To determine plasma ADMA concentration, samples were assayed using a commercially available competitive enzyme immunoassay kit (ADMA-ELISA, DLD Diagnostika GMBH, Hamburg, Germany). As a normal control, ADMA levels were determined in plasma from 12 healthy volunteers (mean age $=35$ years). Markers of endothelial activation, (intracellular adhesion molecule-1 [iCAM-1] and vascular cell adhesion molecule-1 [vCAM-1]) and the inflammatory markers interleukine 6 (IL-6), interleukine 8 (IL-8) all were quantified in duplicates using a bead-based multiplex assay (Bioplex; Bio-Rad Laboratories, Hercules, Calif). Final plasma concentrations were calculated using the Bioplex software supplied by the manufacturer.

Measurement of peripheral vasodilator function. Peripheral vasodilator function was assessed with noninvasive digital pulse amplitude tonometry using the EndoPAT 2000 (Itamar Medical Ltd, Caesarea, Israel). This device provides user-independent measurements of the vasodilator function during reactive hyperemia with a fingertip plethysmograph measuring pulsatile blood volume changes in digital microvessels reflecting the peripheral arterial tone. This method is shown to correlate with flow mediated dilatation of the brachial artery. ${ }^{23,24}$ Measurements were performed bedside at study inclusion and for the next 3 consecutive days with patients in a supine position. Reactive hyperemia was induced by a 5 -min occlusion of the upper arm at approximately $60 \mathrm{mmHg}$ higher than systolic blood pressure. Both sides were measured simultaneously to allow for adjustments of systemic changes in arterial tone by correcting for changes in the nonoccluded arm. Results were analyzed using automated analysis software (EndoPAT 2000 software version 3.1.2) that calculates a ratio between baseline and hyperemic pulsatile volume changes presented as a reactive hyperemia index (RH-index). The RH-index was measured in 10 healthy volunteers with a mean RHindex $2.28(\mathrm{SD} \pm 0.42)$. Previous studies on healthy subjects have reported mean RH-index values around 1.9 to $2 . .^{23,25,26}$

Statistics. Continuous variables are presented as median with interquartile range (IQR). $\chi^{2}$ statistics were used to compare categorical variables. Student $t$ test was used to compare variables with normal distribution, whereas Mann-Whitney $U$ test was used for nonnormally distributed variables. Changes in RHindex and plasma levels of ADMA, IL-6, IL-8, iCAM, and VCAM through the observation period were analyzed using a linear mixed model. All variables needed transformation before the statistical analysis. A linear mixed model also was used to examine for associations among changes in RH-index, ADMA levels with the concomitant day-to-day changes in MAP, and laboratory variables as covariates. Correlations between baseline variables were assessed with Spearman's ranked correlation coefficient $\left(r_{\mathrm{s}}\right)$ or Pearson's correlation coefficient $(r)$. A 2-tailed $P$ value $<0.05$ was considered statistically significant. The SPSS 16.0 software was used for all statistical analysis (Chicago, Ill).

\section{RESULTS}

Study population. Patient characteristics including prior medical history, precipitating factors for acute heart failure, and in hospital procedures are presented in Table I. Most patients with CS presented with acute myocardial infarction and were treated with early percutaneous coronary interventions. Two patients in 
this group had coronary bypass surgery during hospitalization. Treatment with intra-aortic balloon pumps and inotropes/vasopressors were applied in most patients. The open-heart surgical procedures prior to PC-HF included 4 isolated coronary artery bypass grafting (CABG) procedures, 4 aortic valve replacements (2 redo procedures), 1 mitral valve replacement and CABG, 1 composite graft replacement in the ascending aorta and $\mathrm{CABG}, 1$ procedure on the thoracic aorta, and 1 redo closure of left ventricular rupture. No significant differences were found between the 2 groups with regard to the need for inotropic/ vasopressor support. However, the duration of IABP support tended to be longer for CS patients $(P=0.057)$.

Baseline hemodynamic variables and laboratory variables are presented in Table II. Mean time from the onset of acute heart failure to the initial blood sampling and endothelial function measurement was, respectively, 17 and $19 \mathrm{~h}$. CS patients showed on average more severe derangements in their blood gas at baseline suggesting a more profound hypoperfusion in this group. This coincided with significantly lower diuresis and systolic blood pressure.

Markers of inflammation and endothelial activation. Circulating levels of IL-6, IL-8, iCAM-1, and vCAM-1 were not different at baseline between the 2 groups (Table II). IL-6 levels decreased from baseline through day 4 for both CS $(P=0.013)$ and PC-HF $(P<0.001)$ and was significantly lower on days 3 and 4 compared with baseline (Fig 3, A). Similar longitudinal changes were observed for IL-8 levels with a significant decrease for both CS $(P=0.004)$ and PC-HF $(P<0.001)$ (Fig 3, B). IL-6 was negatively correlated with MAP in both CS $\left(r_{\mathrm{s}}=-.57, P<0.001\right)$ and PC-HF $\left(r_{\mathrm{s}}=-.567, P<0.001\right)$. Increased IL-6 levels at baseline also were associated with increased need for vasopressors in CS patients $\left(r_{\mathrm{s}}=.65, P=0.02\right)$.

Circulating levels of ICAM and vCAM did not change from days 1 to 4 for any of the groups (Fig 3, $C$ and $D$ ). ICAM and vCAM were correlated in both groups $\left(\mathrm{CS} r_{\mathrm{s}}=.66, P<0.0001\right.$, PC-HF $r_{\mathrm{s}}=.73$, $P<0.0001)$.

Digital vasodilator function. Satisfactory measurements were obtained in 86 out of 94 potential recordings. Poor patient cooperation and failure to receive a readable digital pulse signal were the main reasons for missing data. The measured RH-index at baseline (day 1) was significantly attenuated compared with healthy controls (RH-index, 2.28) for both CS (RH-index 1.35, $P=$ $0.001)$ and PC-HF (RH-index 1.45, $P=0.001)$. The baseline RH-index was not significantly different between CS and PC-HF (Table II). Also, no significant changes were noted in RH-index for days 1 through 4 for CS patients (Fig 1, $A, P=0.521$ ). In patients with PC-HF, the RH-index changed significantly through the observation period $(P=0.019)$ and was increased at days 3 and 4 compared with baseline (Fig 1, A, $P=$ 0.011). The individual repeated measurements for both groups are displayed in Figures 1, $B$ and $C$ and illustrate the variability among patients both at baseline and during the course of their illness. At baseline, RH-index positively correlated with MAP in PC-HF patients $\left(r_{\mathrm{s}}=\right.$ $.70, P=0.017)$. This association also was present when all recordings were pooled $\left(r_{\mathrm{s}}=.55, P<\right.$ 0.0001). RH-index also correlated to ADMA levels at baseline $\left(r_{\mathrm{s}}=-.633, P=0.036\right)$ for CS patients but not for PC-HF patients. No correlation was found among the measured RH-index and systolic blood pressure, IS, or vasopressor requirements at baseline.

A linear mixed effect model was used to examine for associations among day-to-day changes in RH-index; the concomitant changes in MAP; and plasma levels of ADMA, IL-6, IL-8, iCAM-1, vCAM-1. In patients with PC-HF, increasing MAP and decreasing levels of IL-6 and IL-8 was associated with improvement in RH-index. The only significant correlate for improved RH-index in CS patients was decreasing levels of vCAM-1. We could not detect a significant association between the longitudinal changes in RH-index and ADMA levels in either of the groups.

ADMA. The measured plasma levels of ADMA are shown in Figure 2. Baseline levels of ADMA in CS were similar to those measured in healthy volunteers (0.74 vs $0.79 \mu \mathrm{mol} / \mathrm{L}, P=0.67)$ but significantly elevated compared with PC-HF $(0.55 \mu \mathrm{mol} / \mathrm{L}, P=$ 0.04). A group difference also was found in the course of ADMA levels from baseline through day 4 (Fig 2). ADMA levels increased in patients with PC-HF $(P=$ $0.001)$ and was significantly elevated compared with baseline at day $3(P=0.017)$ and at day $4(P=$ $0.001)$. No significant longitudinal changes were observed for CS patients $(P=0.58)$. Repeating the analysis excluding patients receiving hemodialysis or hemofiltration (2 patients in CS group) did not change these relations. However, a significant positive association was found between the longitudinal changes in ADMA and creatinine $(P=0.032)$ through the observation period. Patients who underwent cardiopulmonary resuscitation prior to CS onset had significantly higher baseline ADMA levels (1.05 vs $0.58 \mu \mathrm{mol} / \mathrm{L}, P=0.003)$. In CS patients, baseline ADMA levels correlated with both lactate measured at baseline $\left(r_{\mathrm{s}}=.85, P=0.001\right)$ and the maximal lactate measured within $48 \mathrm{~h}\left(r_{\mathrm{s}}=.90, P<0.001\right)$. This association was not present in PC-HF patients. No correlations were found between baseline ADMA 
Table I. Study population. Baseline characteristics, precipitating factors, and in hospital treatment and outcomes

\begin{tabular}{|c|c|c|}
\hline & $\operatorname{CS}(n=12)$ & $P C-H F^{*}(n=12)$ \\
\hline Age, years (mean,range) & $65.8(49-85)$ & 70.7 (58-79) \\
\hline Male gender, $\mathrm{n}$ & 8 & 10 \\
\hline \multicolumn{3}{|l|}{ Prior medical history, n } \\
\hline Coronary heart disease & 5 & 7 \\
\hline Myocardial infarction & 5 & 4 \\
\hline Chronic heart failure & 3 & 3 \\
\hline Hypertension & 8 & $2^{*}$ \\
\hline Severe valvular disease & 0 & 6 \\
\hline Diabetes & 3 & 4 \\
\hline Stroke/TIA & 3 & 0 \\
\hline Renal failure & 1 & 0 \\
\hline Chronic obstructive pulmonary disease & 3 & 0 \\
\hline Current smoking & 4 & 3 \\
\hline \multicolumn{3}{|l|}{ Precipitating factors, $\mathrm{n}$} \\
\hline Acute coronary syndromes & 11 & \\
\hline STEMI & 7 & \\
\hline Non-STEMI & 4 & \\
\hline Arrhythmias & 1 & \\
\hline $\mathrm{CPR}^{\boldsymbol{\pi}}$ & 5 & 2 \\
\hline \multicolumn{3}{|l|}{ Treatment and procedures } \\
\hline $\mathrm{PCl}, \mathrm{n}$ & 9 & \\
\hline CABG, $\mathrm{n}$ & 2 & \\
\hline Other heart surgery, $\mathrm{n}$ & 0 & \\
\hline Mechanical ventilation, $\mathrm{n}$ & 7 & $8^{\dagger}$ \\
\hline Pulmonary artery catheter, $\mathrm{n}$ & 6 & 3 \\
\hline Ventricular assist device, $\mathrm{n}$ & 2 & 1 \\
\hline IABP, $n$ & 11 & 10 \\
\hline Duration of IABP support, $h$ & $167(65-240)$ & $55(39-180)$ \\
\hline Inotropes, n & 11 & 11 \\
\hline Vasopressors, n & 10 & 12 \\
\hline Duration of vasopressor/inotropic support, $\mathrm{h}$ & $84(55-148)$ & $51(24-94)$ \\
\hline Maximal vasopressor dose $e^{\ddagger, \S}$ & $0.10(0.01-0.28)$ & $0.13(0.07-0.21)$ \\
\hline Maximal IS ${ }^{\ddagger}$ & $18.5(5.2-31.0)$ & $17.2(12.4-30.0)$ \\
\hline Hemofiltration or hemodialysis, $\mathrm{n}$ & 2 & 0 \\
\hline Length of ICU/CCU stay, days & $10(5-17)$ & $7(3-14)$ \\
\hline Maximal SOFA score* & $7.5(6.0-14.8)$ & $8.5(5.3-11.5)$ \\
\hline Organ failure, ${ }^{+\dagger} \mathrm{n}$ & 5 & 6 \\
\hline In hospital mortality, $\mathrm{n}$ & 5 & 2 \\
\hline
\end{tabular}

EQ1 Continuous variables are presented as median with $I Q R$ if not stated otherwise.

$\mathrm{PCl}$, percutaneous coronary intervention; TIA, transitory ischemic attack.

*Surgical procedures are presented under methods.

${ }^{\dagger}$ Patients necessitating intubation exceeding the first postoperative day.

${ }^{\ddagger}$ Measured within the first $48 \mathrm{~h}$.

$\S$ Given as the total dose of epinephrine and norepinephrine (ug/kg/min).

"Cardiopulmonary resuscitation prior to inclusion.

**Total seguential organ failure assessment score (SOFA) score except neurological SOFA subscore.

${ }^{+\dagger}$ Failure of $\geq 1$ organ other than cardiovascular failure.

$\ddagger \ddagger P<0.05$ compared with $\mathrm{CS}$.

and $17 \%$. However, only 1 fatality occurred during the and the need for vasopressors or IS. Baseline ADMA levels did correlate with baseline MAP for PC-HF patients $\left(r_{\mathrm{s}}=.73, P=0.007\right)$ but not for CS patients. No association between day-to-day changes in ADMA and changes in MAP, IL-6, IL-8, iCAM-1, vCAM-1 could be observed in either group.

In hospital outcome and organ dysfunction. Hospital mortality rates in CS and PC-HF was, respectively, $42 \%$ first 4 days of follow-up (CS group). No significant difference was found in RH-index and baseline levels of ADMA, IL-6, IL-8, iCAM-1, and vCAM-1 between hospital survivors and nonsurvivors. The maximal total SOFA score (excluding neurological subscore) for days 1 through 4 was not significantly different between CS and PC-HF patients (Table I). 
Table II. Baseline laboratory and hemodynamic variables

\begin{tabular}{|c|c|c|}
\hline & $\operatorname{cs} n=12$ & $\mathrm{PC}-\mathrm{HF} \mathrm{n}=12$ \\
\hline CK-mb ( $\mu \mathrm{g} / \mathrm{L})$, maximal value & 207 (47-493) & $56(25-133)$ \\
\hline Plasma creatinine $(\mu \mathrm{mol} / \mathrm{L})$ & $125(99-183)$ & $85(81-121)$ \\
\hline eGFR (mL/min) & $57(40-114)$ & $84(63-102)$ \\
\hline Lactate (mmol/L), Baseline* & $3.3(1.8-7.9)$ & $1.9(1.5-6.2)$ \\
\hline Highest value $^{\dagger}$ & $3.3(1.8-8.4)$ & $5.2(2.1-7.0)$ \\
\hline Arterial $\mathrm{pH}$, Baseline* & $7.26(7.11-7.31)$ & $7.36(7.29-7.43)^{\S}$ \\
\hline Lowest value $^{\dagger}$ & $7.20(7.05-7.30)$ & $7.26(7.13-7.34)$ \\
\hline Base excess(mmol/L), Baseline ${ }^{*}$ & $-7.7(-12.6--3.9)$ & $-4.4(-5.7--1.8)^{\S}$ \\
\hline Lowest value $^{\dagger}$ & $-8.1(-17.8--3.9)$ & $-6.2(-12.5--2.7)$ \\
\hline C-reactive protein (mg/L) & $33(9-135)$ & $69(10-121)$ \\
\hline White blood cells $\left(10^{9} / \mathrm{L}\right)$ & $17.2(9.8-19.8)$ & $9.5(5.4-11.7)^{\S}$ \\
\hline ADMA (umol/L) & $0.62(0.52-1.11)$ & $0.51(0.37-0.68)^{s}$ \\
\hline $\mathrm{IL}-6(\mathrm{pg} / \mathrm{mL})$ & $281(131-459)$ & $342(197-691)$ \\
\hline IL-8 (pg/mL) & $121(26-122)$ & $57(43-140)$ \\
\hline iCAM-1 (ng/mL) & 96 (64-152) & $98(88-269)$ \\
\hline vCAM-1 (ng/mL) & $349(257-441)$ & $367(311-412)$ \\
\hline $\mathrm{RH}$-index & $1.35(1.27-1.56)$ & $1.45(1.00-1.86)$ \\
\hline Mean arterial blood pressure (mmHG) & $60(56-67)$ & $64(57-68)$ \\
\hline Systolic blood pressure (mmHG) & $80(75-85)$ & $90(80-99)^{\S}$ \\
\hline Diastolic blood pressure (mmHG) & $53(46-58)$ & $50(40-55)$ \\
\hline Cardiac index $\left(\left\llcorner/ \mathrm{min} / \mathrm{m}^{2}\right)^{\ddagger}\right.$ & $2.18(1.51-2.47)$ & \\
\hline SVRI (dynes*sec $\left./ \mathrm{cm}^{5^{*}} \mathrm{~m}^{2}\right)^{\ddagger}$ & $2097(1961-2576)$ & \\
\hline Diuresis, $\mathrm{ml} /$ hour & $10(0-29)$ & $68(25-91)^{\S}$ \\
\hline HR (beats per min) & $110(85-120)$ & 88 (85-99) \\
\hline
\end{tabular}

Values are presented as median with $I Q R$.

$C K-m b$, creatine kinase $m b$; eGFR, estimated glomerular filtration rate; SVRI, systemic vascular resistance index.

*First measurement after onset of CS or PC-HF.

${ }^{\dagger}$ Measured within first $48 \mathrm{~h}$.

${ }^{{ }_{n}} \mathrm{n}=6$. eGFR calculated using the modification of diet in renal disease formula.

$\S p<0.05$ compared with cardiogenic shock.

In CS patients, the SOFA score was correlated to baseline RH-index $\left(r_{\mathrm{s}}=-.69, P=0.014\right)$, baseline ADMA levels $\left(r_{\mathrm{s}}=.630, P=0.028\right)$, baseline IL-6 $\left(r_{\mathrm{s}}=.62, P=0.03\right)$, and IL-8 $\left(r_{\mathrm{s}}=.78, P=0.007\right)$. Furthermore, baseline ADMA levels were strongly correlated to the hepatic $\left(r_{\mathrm{s}}=.79, P=0.003\right)$ and respiratory $\left(r_{\mathrm{s}}=.77, P=0.004\right)$ SOFA subscores, whereas no significant associations were observed for the other organs including the kidneys $\left(r_{\mathrm{s}}=.55, P=\right.$ $0.065)$. ADMA levels increased in 7 patients and decreased in 5 patients through the observation period. A negative correlation existed between the absolute change in ADMA levels and the degree of hepatic and respiratory dysfunction $\left(r_{\mathrm{s}}=-.63, P=0.024\right.$ and $r_{\mathrm{s}}=-.71, P=0.01$, respectively). CS patients with failure of an organ other than the central nervous system or cardiovascular system had lower baseline RH-index (median 1.22 vs $1.51, P=0.01$ ), elevated ADMA levels (median 0.58 vs $1.05 \mathrm{umol} / \mathrm{L}, P=0.005$ ), and increased IL-8 (median $27 \mathrm{vs.} 150 \mathrm{pg} / \mathrm{mL}$ ) compared with patients without organ failure. A tendency also was noted toward increased IL-6 levels in CS patients with organ failure, but this finding was not statistically significant (132 vs $343 \mathrm{pg} / \mathrm{mL}, P=0.202$ ).
For patients with PC-HF, no association was found among SOFA scores and baseline RH-index, ADMA, or IL-6. IL-8 did, however, correlate to SOFA score $\left(r_{\mathrm{s}}=.79, P=0.002\right)$. Accordingly, baseline RHindex, ADMA, IL-8, and IL-6 were not different in patients with organ failure compared with those without. Also, no correlation was found between the change in ADMA levels and any of the SOFA subscores. No association was evident among iCAM-1, vCAM-1, and SOFA scores in any group.

\section{DISCUSSION}

Our study shows that patients with the most severe forms of acute heart failure (ie, CS and PC-HF) are subject to a profound inflammatory insult evident by excessive circulating levels of IL-6 and IL-8, markedly higher than reported in acute decompensated heart failure and acute myocardial infarction. ${ }^{9,10,27}$ These levels are comparable with the levels measured in sepsis. ${ }^{7,28,29}$ This finding was accompanied by a sustained elevation in markers of endothelial activation. Although the systemic inflammatory insult and endothelial activation inflicted by open-heart surgery and use of extra corporeal 
647

648

649

650

651

652

653

654

655

656

657

658

659

660

661

662

663

664

665

666

667

668

669

670

671

672

673

674

675

676

677

678

679

680

681

682

683

684

685

686

687

688

689

690

691

692

693

694

695

696

697

698

699

700

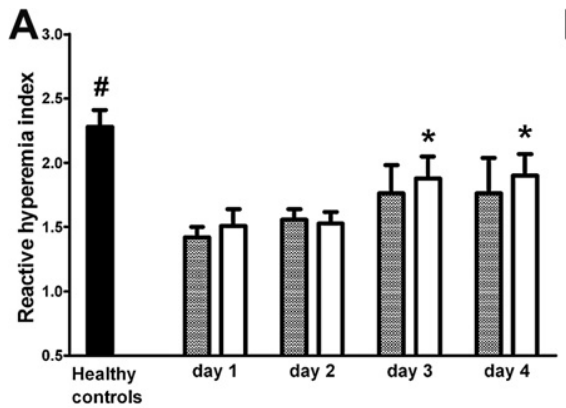

Cardiogenic shock

$\square$ Postcardiotomy heart failure

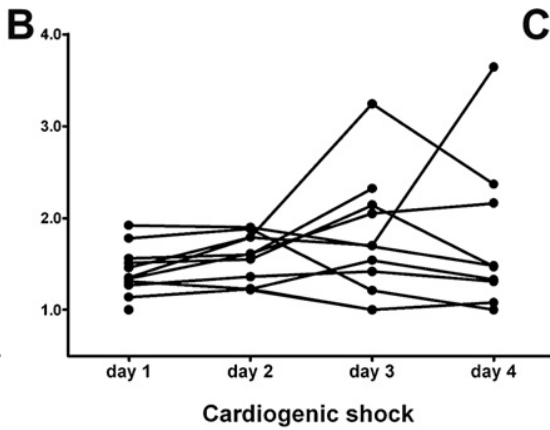

Cardiogenic shock

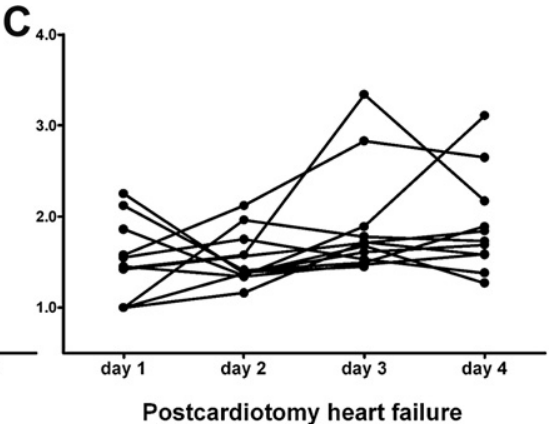

Postcardiotomy heart failure

Fig 1. RH-index measured at days 1-4. A, Mean RH-index with SEM. Individual values of the repeated RH-index measurements for CS patients B, and PC-HF patients C. One patient in the CS group died at day 2 . $* P<0.05$ compared with day 1. ${ }^{\#} P<0.05$ compared with $\mathrm{CS}$ and PC-HF at day 1.
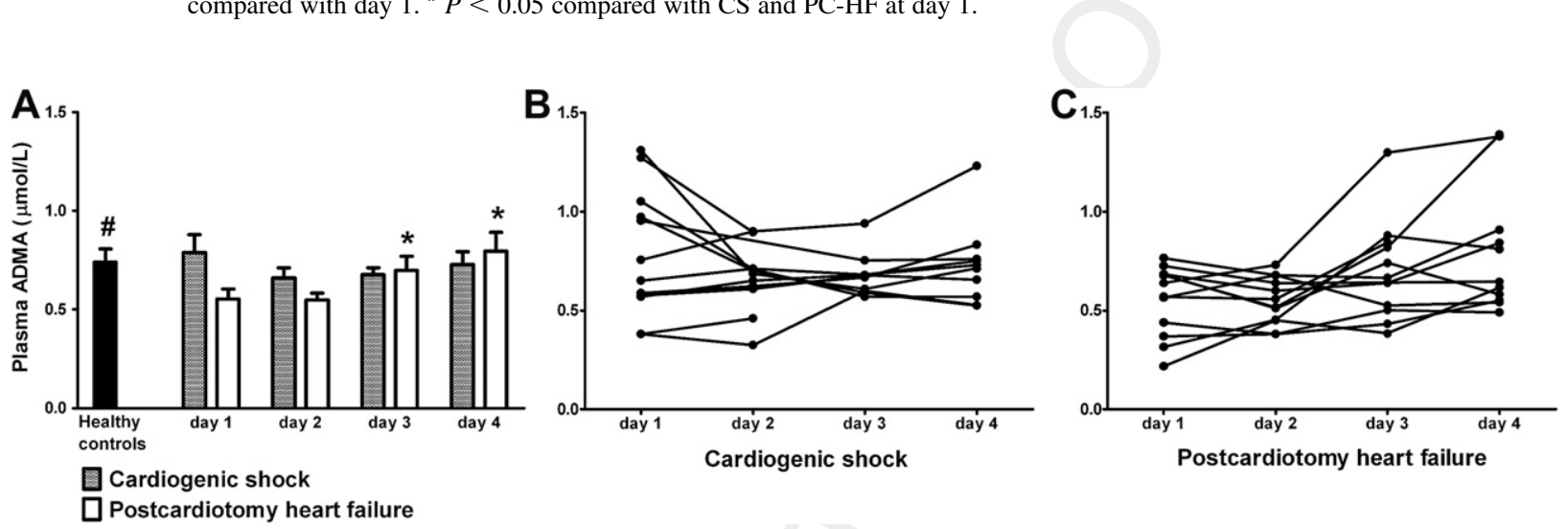

Fig 2. The course of plasma ADMA levels measured at days 1-4. A, Mean plasma ADMA with SEM. Individual changes in plasma ADMA levels for CS (A) and PC-HF patients (B). One patient in the cardiogenic shock group died at day 2 . $* P<0.05$ compared with day $1 .{ }^{*} P<0.05$ compared with PC-HF at day 1 .

bypass is well described, ${ }^{30}$ the cause and consequence of this in CS is less well understood. However, the previously described association between IL-6 levels and vasopressor requirements to restore blood pressure in CS also was evident in our study as high IL-6 levels were both related to low MAP and increased vasopressor dose. ${ }^{6}$

The peripheral vasodilator function was assessed serially through the first 4 days after acute heart failure onset. The RH-index was impaired at baseline in both groups compared with healthy controls. This finding was, however, not uniform as several individuals had a preserved RH-index indicating a preserved vascular and endothelial function. A few prior studies have assessed vascular reactivity in shock and critical illness demonstrating impaired vascular response during reactive hyperemia. Kirschenbaum et al found an attenuated increase in forearm blood flow after reactive hyperemia in patients with cardiogenic and septic shock compared with healthy controls. ${ }^{31}$ A similar observation was made in patients with severe sepsis using peripheral arterial tonometry similar to the present study. ${ }^{29}$ The repeated assessments in our study also revealed a substantial individual day-to-day variation. This finding underlines that vascular function and probably endothelial function in acute disease are not static, which is an important consideration to make when assessing vascular function in acute versus chronic disease states.

The mechanisms behind the apparent attenuation in vasodilator function and the changes observed through the course of the disease are likely to be multifactorial and probably a composite of prior vascular/endothelial function and a multitude of superimposed acute factors. The peripheral vasculature in CS and PC-HF is subject to an immense and changing stimulus from circulating hormones, the sympathetic nervous system, and local metabolic factors capable of modulating both endothelial and vascular function. ${ }^{32-34}$ Also, fewer functional capillaries secondary to intravascular obstruction or oedema could blunt the reactive hyperemic response in critical disease. ${ }^{35}$ The RH-index, as a measure of the peripheral circulations ability to respond to transitory local ischemia, is likely a result from all these factors. Being in part NO-dependent, the impaired RH-index 

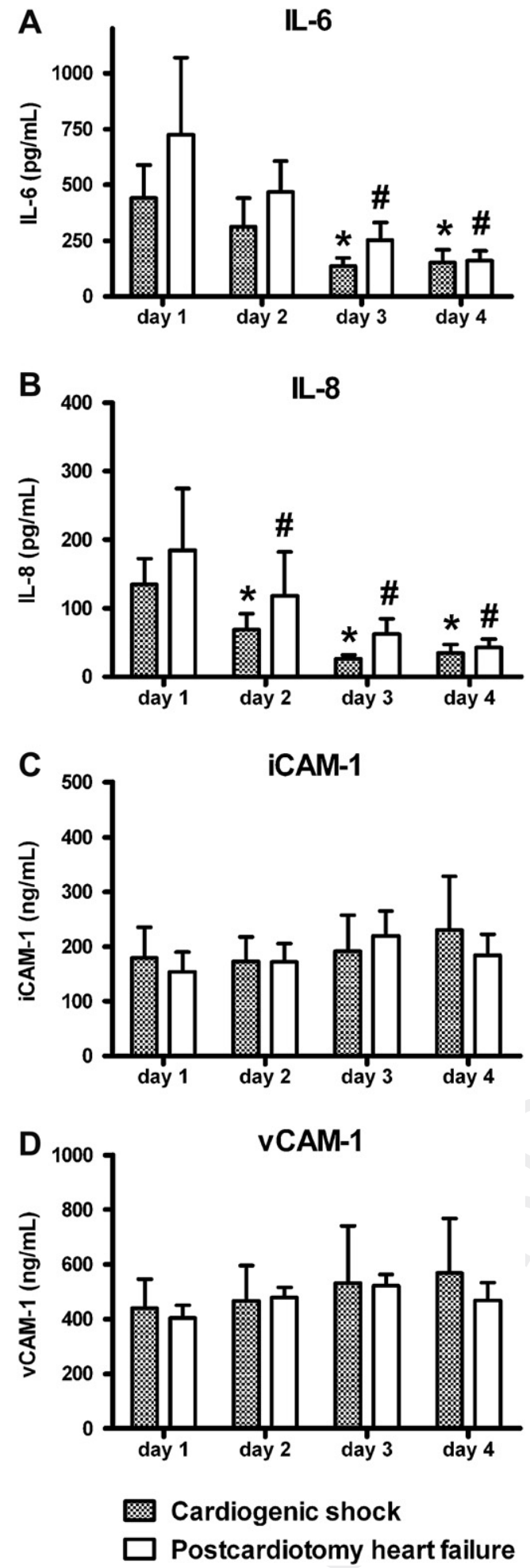

Fig 3. A, Circulating plasma levels of IL-6. B, IL-8. C, iCAM-1. D, vCAM-1. One patient in the CS group died at day 2 . ${ }^{*} P<0.05 \mathrm{com}-$ pared with day 1 for $\mathrm{CS}$ patients. ${ }^{\#} P<0.05$ compared with day 1 for PC-HF patients. at baseline could suggest a reduced endothelial NO bioavailability. ${ }^{25}$ The negative relation observed between the endogenous NO-inhibitor ADMA and the RHindex at baseline in CS could suggest an inhibitory effect of ADMA on vasodilator function. However, no longitudinal association was found between the two to back up this hypothesis. Also, this relation was not observed in PC-HF. It is possible that attenuation of other important contributors to endothelium dependent dilation, such as prostacyclin or endothelium derived hyperpolarizing factor, play a bigger role. Interestingly, the observed improvement in vasodilator function in $\mathrm{PC}-\mathrm{HF}$ was associated with a concomitant decrease in inflammatory cytokines. A transitory impairment of endothelial function after exposure to cytokines and transitory inflammation has been described in an experimental setting and possibly could explain the effects observed here. ${ }^{36,37}$ Furthermore, tumor necrosis factor $\alpha(\mathrm{TNF} \alpha)$ and IL-6 also have been shown to induce endothelium-dependent vasoconstriction in human arterial segments. ${ }^{38}$

Role of ADMA in CS and PC-HF. Circulating ADMA levels were similar in CS and healthy controls. The normal values found in this study coincide well with a recently proposed normal reference value (mean 0.69 , 95\% confidence interval: $0.36-1.17 \mu \mathrm{mol} / \mathrm{L}$ ) and other reports using a similar enzyme-linked immunosorbent assay technique. ${ }^{39,40}$ This is in contrast to previous reports measuring elevated levels of ADMA after acute decompensated heart failure and CS using highperformance liquid chromatography and mass spectrometry, respectively. ${ }^{16,18}$ A significant elevation was, however, evident in the most critically ill CS patients in our cohort with high arterial lactate and organ failure

Baseline ADMA levels were suppressed in PC-HF, and although no pattern of change was apparent during the first 4 days in CS, ADMA tended to increase in PCHF patients. This could possibly be caused by a hemodiluting effect of extra corporeal circulation, as initially suppressed postoperative ADMA levels previously have been demonstrated in patients undergoing openheart surgery. ${ }^{41,42}$ This consideration is important to make when using ADMA as a risk marker in critically ill patients in whom surgery has been done. The relatively suppressed ADMA levels in PC-HF also could reflect that, in our population of patients, this condition is more benign and that most of these patients were in a state of recovery as compared with CS. However, no indication was given that the degree or course of inflammation and endothelial activation were related to ADMA levels. Except for the observed correlation with MAP at baseline in PC-HF, ADMA levels were 
not related to blood pressure or vasopressor/inotrope requirements.

Elevated ADMA levels were associated with the overall degree of organ dysfunction (SOFA score) and elevated systemic lactate in CS. Accordingly, CS patients with organ failure had significantly higher ADMA levels. In particular, this elevation was related to the degree of respiratory and hepatic dysfunction. Although baseline ADMA levels were not significantly correlated with the renal SOFA score, the longitudinal positive association between ADMA and creatinine were consistent with prior reports that ADMA accumulates secondary to renal dysfunction. ${ }^{43}$ These observations are in line with findings presented in previous reports from patients with severe sepsis and in critically ill patients with organ failure. ${ }^{17,44,45}$ Accumulation of ADMA is thought to occur mainly through reduced metabolism secondary to dysregulation and inhibition of the dimethylarginine dimethylaminohydrolase (DDAH) ${ }^{46}$ This enzyme is highly expressed in both liver and kidneys, which are presumed to be the main routs for metabolic clearance of circulating ADMA. However, ADMA levels were highest in patients receiving cardiopulmonary resuscitation (CPR) either before or at the onset of shock. It is likely that these patients had the most profound systemic hypoperfusion. Unlike septic patients, the observed early elevated lactate levels in CS patients are likely a result of systemic hypoperfusion rather than liver failure. It is from the available data not possible to determine whether patients in shock already have accumulated levels of ADMA or if they develop this after shock onset secondary to acute reduction in hepatic or renal elimination. Increased ADMA levels in patients who had CPR and/or had elevated arterial lactate could indicate that ADMA as a marker of elevated risk in CS merely reflects the severity of hypoperfusion during shock. However, ADMA also could potentially play a causative role in hypoperfusion and development of organ failure through inhibiting the NO-mediated regulation of organ blood flow. ${ }^{47}$

Although ADMA was increased in patients with failing organs compared with those without, these patients did not accumulate more ADMA during the observation period. On the contrary, ADMA decreased more in patients with the highest degree of hepatic dysfunction.

Our study did not support an association between the degree of systemic inflammation and circulating ADMA. In vitro studies on endothelial cells, however, have demonstrated that oxidative stress, stimulation with $\mathrm{TNF} \alpha$, and nitrosative stress resulting from an induction of inducible NO synthase cause an accumulation of ADMA secondary to reduced DDAH activity. ${ }^{48,49}$ However, increased DDAH activity secondary to stimulation with inflammatory cytokine have been reported in rat vascular smooth muscle cells, ${ }^{50}$ and a mechanistic knowledge of the important metabolic responses and the regulation of the NOsystem and vasculature is still warranted.

\section{LIMITATIONS}

The vascular function measurements were performed in a clinical setting in the ICU/CCU on patients receiving several different pharmacological and mechanical treatments through the observation period. This lack of standardization could affect the quality of these measurements. Invasive hemodynamics were only measured in 1/4 of the patients, and thus, no evaluation of $\mathrm{RH}$-index in relation to changing hemodynamics could be performed. Also, multiple statistical comparisons based on a limited number of patients inherits the risk of type I errors.

\section{CONCLUSION}

In this study, both CS and PC-HF were characterized by initially elevated levels of inflammatory cytokines suggestive of a profound inflammatory insult accompanied by an attenuated peripheral vasodilator function. The resolving inflammatory response was associated with improved RH-index in PC-HF patients, whereas no such relation was evident for CS patients. The circulating levels of the endogenous NO-inhibitor ADMA did not relate to the degree of inflammation or to the day-today changes in vasodilator function. However, in CS, ADMA, vasodilator function, and levels of inflammatory cytokines were correlated to the overall degree of organ dysfunction, and to hepatic dysfunction in particular. The increased ADMA levels in patients who had CPR and/or elevated arterial lactate suggest a relation between ADMA and the degree of hypoperfusion in CS.

The authors wish to thank Helse Nord RHF for financing this study.

\section{REFERENCES}

1. Hochman JS. Cardiogenic shock complicating acute myocardial infarction: expanding the paradigm. Circulation 2003;107: 2998-3002.

2. Menon V, Slater JN, White HD, Sleeper LA, Cocke T, Hochman JS. Acute myocardial infarction complicated by systemic hypoperfusion without hypotension: report of the SHOCK trial registry. Am J Med 2000;108:374-80.

3. Kohsaka S, Menon V, Lowe AM, et al. Systemic inflammatory response syndrome after acute myocardial infarction complicated by cardiogenic shock. Arch Intern Med 2005;165:1643-50.

4. Hochman JS, Buller CE, Sleeper LA, et al. Cardiogenic shock complicating acute myocardial infarction-etiologies, management and outcome: a report from the SHOCK Trial Registry. Should we emergently revascularize occluded coronaries for cardiogenic shock? J Am Coll Cardiol 2000;36:1063-70.

5. Alexander JH, Reynolds HR, Stebbins AL, et al. Effect of tilarginine acetate in patients with acute myocardial infarction and cardiogenic shock: the TRIUMPH randomized controlled trial. JAMA 2007;297:1657-66. 
6. Geppert A, Dorninger A, Delle-Karth G, Zorn G, Heinz G, Huber K. Plasma concentrations of interleukin-6, organ failure, vasopressor support, and successful coronary revascularization in predicting 30-day mortality of patients with cardiogenic shock complicating acute myocardial infarction. Crit Care Med 2006; 34:2035-42.

7. Geppert A, Steiner A, Zorn G, et al. Multiple organ failure in patients with cardiogenic shock is associated with high plasma levels of interleukin-6. Crit Care Med 2002;30:1987-94.

8. Katayama T, Nakashima H, Takagi C, Honda Y, Suzuki S, Yano K. Predictors of mortality in patients with acute myocardial infarction and cardiogenic shock. Circ J 2005;69:83-8.

9. Theroux P, Armstrong PW, Mahaffey KW, et al. Prognostic significance of blood markers of inflammation in patients with STsegment elevation myocardial infarction undergoing primary angioplasty and effects of pexelizumab, a C5 inhibitor: a substudy of the COMMA trial. Eur Heart J 2005;26:1964-70.

10. Debrunner M, Schuiki E, Minder E, et al. Proinflammatory cytokines in acute myocardial infarction with and without cardiogenic shock. Clin Res Cardiol 2008;97:298-305.

11. Boger RH. Asymmetric dimethylarginine (ADMA): a novel risk marker in cardiovascular medicine and beyond. Ann Med 2006; 38:126-36.

12. Stuhlinger MC, Oka RK, Graf EE, et al. Endothelial dysfunction induced by hyperhomocyst(e)inemia: role of asymmetric dimethylarginine. Circulation 2003;108:933-8.

13. Ohnishi M, Wada A, Tsutamoto T, et al. Endothelin stimulates an endogenous nitric oxide synthase inhibitor, asymmetric dimethylarginine, in experimental heart failure. Clin Sci (Lond) 2002;103 (Suppl. 48):241S-4S.

14. Leiper J, Nandi M, Torondel B, et al. Disruption of methylarginine metabolism impairs vascular homeostasis. Nat Med 2007;13: 198-203.

15. Achan V, Broadhead M, Malaki M, et al. Asymmetric dimethylarginine causes hypertension and cardiac dysfunction in humans and is actively metabolized by dimethylarginine dimethylaminohydrolase. Arterioscler Thromb Vasc Biol 2003;23:1455-9.

16. Nicholls SJ, Wang Z, Koeth R, et al. Metabolic profiling of arginine and nitric oxide pathways predicts hemodynamic abnormalities and mortality in patients with cardiogenic shock after acute myocardial infarction. Circulation 2007;116:2315-24.

17. Nijveldt RJ, Teerlink T, Van Der Hoven B, et al. Asymmetrical dimethylarginine (ADMA) in critically ill patients: high plasma ADMA concentration is an independent risk factor of ICU mortality. Clin Nutr 2003;22:23-30.

18. Saitoh M, Osanai T, Kamada T, et al. High plasma level of asymmetric dimethylarginine in patients with acutely exacerbated congestive heart failure: role in reduction of plasma nitric oxide level. Heart Vessels 2003;18:177-82.

19. Duckelmann C, Mittermayer F, Haider DG, Altenberger J, Wolzt M. Plasma asymmetric dimethylarginine and cardiovascular events in patients with acute decompensated heart failure. Trans Res 2008;152:24-30.

20. Wernovsky G, Wypij D, Jonas RA, et al. Postoperative course and hemodynamic profile after the arterial switch operation in neonates and infants. A comparison of low-flow cardiopulmonary bypass and circulatory arrest. Circulation 1995;92:2226-35.

21. Chen JS, Ko WJ, Yu HY, et al. Analysis of the outcome for patients experiencing myocardial infarction and cardiopulmonary resuscitation refractory to conventional therapies necessitating extracorporeal life support rescue. Crit Care Med 2006;34:950-7.

22. Vincent JL, Moreno R, Takala J, et al. The SOFA (Sepsis-related Organ Failure Assessment) score to describe organ dysfunction/ failure. On behalf of the Working Group on Sepsis-Related Prob- lems of the European Society of Intensive Care Medicine. Intensive Care Med 1996;22:707-10.

23. Dhindsa M, Sommerlad SM, DeVan AE, et al. Interrelationships among noninvasive measures of postischemic macro- and microvascular reactivity. J Appl Physiol 2008;105:427-32.

24. Kuvin JT, Patel AR, Sliney KA, et al. Assessment of peripheral vascular endothelial function with finger arterial pulse wave amplitude. Am Heart J 2003;146:168-74.

25. Nohria A, Gerhard-Herman M, Creager MA, Hurley S, Mitra D, Ganz P. Role of nitric oxide in the regulation of digital pulse volume amplitude in humans. J Appl Physiol 2006;101:545-8.

26. Yeo TW, Lampah DA, Gitawati R, et al. Impaired nitric oxide bioavailability and L-arginine reversible endothelial dysfunction in adults with falciparum malaria. J Exp Med 2007;204:2693-704.

27. Matsumoto M, Tsujino T, Lee-Kawabata $M$, et al. Serum interleukin-6 and C-reactive protein are markedly elevated in acute decompensated heart failure patients with left ventricular systolic dysfunction. Cytokine 2010;49:264-8.

28. de Werra I, Jaccard C, Corradin SB, et al. Cytokines, nitrite/nitrate, soluble tumor necrosis factor receptors, and procalcitonin concentrations: comparisons in patients with septic shock, cardiogenic shock, and bacterial pneumonia. Crit Care Med 1997;25: 607-13.

29. Davis JS, Yeo TW, Thomas JH, et al. Sepsis-associated microvascular dysfunction measured by peripheral arterial tonometry: an observational study. Crit Care 2009;13:R155.

30. Paparella D, Yau TM, Young E. Cardiopulmonary bypass induced inflammation: pathophysiology and treatment. An update. Eur J Cardiothorac Surg 2002;21:232-44.

31. Kirschenbaum LA, Astiz ME, Rackow EC, Saha DC, Lin R. Microvascular response in patients with cardiogenic shock. Crit Care Med 2000;28:1290-4.

32. Thijssen DH, de Groot PC, Kooijman M, Smits P, Hopman MT. The sympathetic nervous system contributes to the age-related impairment of flow-mediated dilation of the superficial femoral artery. Am J Physiol Heart Circ Physiol 2006;291:H3122-9.

33. Berger R, Stanek B, Hulsmann M, et al. Effects of endothelin a receptor blockade on endothelial function in patients with chronic heart failure. Circulation 2001;103:981-6.

34. Quayle JM, Nelson MT, Standen NB. ATP-sensitive and inwardly rectifying potassium channels in smooth muscle. Physiol Rev 1997;77:1165-232.

35. Astiz ME, DeGent GE, Lin RY, Rackow EC. Microvascular function and rheologic changes in hyperdynamic sepsis. Crit Care Med 1995;23:265-71.

36. Clapp BR, Hingorani AD, Kharbanda RK, et al. Inflammationinduced endothelial dysfunction involves reduced nitric oxide bioavailability and increased oxidant stress. Cardiovasc Res 2004;64:172-8.

37. Hingorani AD, Cross J, Kharbanda RK, et al. Acute systemic inflammation impairs endothelium-dependent dilatation in humans. Circulation 2000;102:994-9.

38. Iversen PO, Nicolaysen A, Kvernebo K, Benestad HB, Nicolaysen G. Human cytokines modulate arterial vascular tone via endothelial receptors. Pflugers Arch 1999;439:93-100.

39. Schulze F, Maas R, Freese R, Schwedhelm E, Silberhorn E, Boger RH. Determination of a reference value for $N(G), N$ (G)-dimethyl-L-arginine in 500 subjects. Eur J Clin Invest 2005;35:622-6.

40. Horowitz JD, Heresztyn T. An overview of plasma concentrations of asymmetric dimethylarginine (ADMA) in health and disease and in clinical studies: methodological considerations. J Chromatogr B Analyt Technol Biomed Life Sci 2007;851:42-50. 
41. Karu I, Zilmer K, Starkopf J, Zilmer M. Changes of plasma asymmetric dimethylarginine levels after coronary artery bypass grafting. Scand Cardiovasc J 2006;40:363-7.

42. Loukanov T, Arnold R, Gross J, et al. Endothelin-1 and asymmetric dimethylarginine in children with left-to-right shunt after intracardiac repair. Clin Res Cardiol 2008;97:383-8.

43. Vallance P, Leone A, Calver A, Collier J, Moncada S. Accumulation of an endogenous inhibitor of nitric oxide synthesis in chronic renal failure. Lancet 1992;339:572-5.

44. O’Dwyer MJ, Dempsey F, Crowley V, Leher DP, McManus R, Ryan T. Septic shock is correlated with asymmetrical dimethyl arginine levels, which may be influenced by a polymorphism in the dimethylarginine dimethylaminohydrolase II gene: a prospective observational study. Crit Care 2006;10:R139.

45. Richir MC, Ellger B, Teerlink T, et al. The effect of rosiglitazone on asymmetric dimethylarginine (ADMA) in critically ill patients. Pharmacol Res 2009;60:519-24.
46. Teerlink T. ADMA metabolism and clearance. Vasc Med 2005;10 (Suppl. 1):S73-81.

47. Nijveldt RJ, Teerlink T, van Leeuwen PA. The asymmetrical dimethylarginine (ADMA)-multiple organ failure hypothesis. Clin Nutr 2003;22:99-104.

48. Ito A, Tsao PS, Adimoolam S, Kimoto M, Ogawa T, Cooke JP. Novel mechanism for endothelial dysfunction: dysregulation of dimethylarginine dimethylaminohydrolase. Circulation 1999;99: 3092-5.

49. Leiper J, Murray-Rust J, McDonald N, Vallance P. S-nitrosylation of dimethylarginine dimethylaminohydrolase regulates enzyme activity: further interactions between nitric oxide synthase and dimethylarginine dimethylaminohydrolase. Proc Natl Acad Sci U S A 2002; 99:13527-32.

50. Ueda S, Kato S, Matsuoka H, et al. Regulation of cytokineinduced nitric oxide synthesis by asymmetric dimethylarginine: role of dimethylarginine dimethylaminohydrolase. Circ Res 2003;92:226-33. 


\section{AUTHOR QUERY FORM}

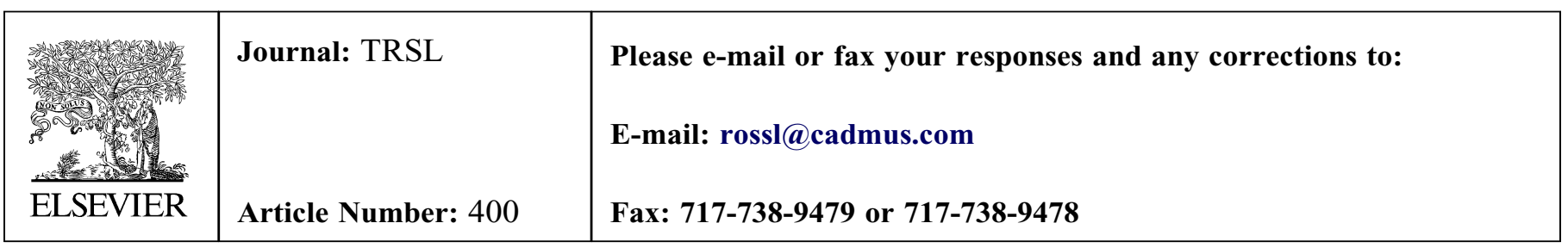

Dear Author,

Please check your proof carefully and mark all corrections at the appropriate place in the proof (e.g., by using on-screen annotation in the PDF file) or compile them in a separate list.

For correction or revision of any artwork, please consult http://www.elsevier.com/artworkinstructions.

Any queries or remarks that have arisen during the processing of your manuscript are listed below and highlighted by flags in the proof.

\begin{tabular}{|c|c|}
\hline $\begin{array}{c}\text { Location } \\
\text { in article }\end{array}$ & \multicolumn{1}{c|}{$\begin{array}{c}\text { Query / Remark: Click on the Q link to find the query's location in text } \\
\text { Please insert your reply or correction at the corresponding line in the proof }\end{array}$} \\
\hline Q1 & Please cite figures in numerical order. Figure 3 should come after figure 1 and 2. \\
EQ1 & The $+\$$ dagger occured in table footnote but not cited in table body. Please provide the citation. \\
\hline
\end{tabular}

Thank you for your assistance. 